\title{
Review
}

Journal of Innate

Immunity
$\mathrm{J}$ Innate Immun 2011;3:365-373

DOI: $\underline{10.1159 / 000328505}$
Received: November 1, 2010

Accepted after revision: April 19, 2011

Published online: May 11, 2011

\section{NK Cell Recognition and Killing of Melanoma Cells Is Controlled by Multiple Activating Receptor-Ligand Interactions}

\author{
Sara Morgado ${ }^{a}$ Beatriz Sanchez-Correa ${ }^{a}$ Javier G. Casado ${ }^{a}$ Esther Duran ${ }^{b}$ \\ Inmaculada Gayoso ${ }^{c}$ Fernando Labella ${ }^{d}$ Rafael Solana ${ }^{c}$ Raquel Tarazona ${ }^{a}$ \\ a Immunology Unit, Department of Physiology, and ${ }^{b}$ Comparative Pathology Unit, Department of Animal Medicine, \\ University of Extremadura, Cáceres, and 'Immunology Unit, Faculty of Medicine, University of Córdoba, Hospital \\ Reina Sofía, Instituto Maimónides para la Investigación Biomédica de Córdoba (IMIBIC), and dOphthalmology Unit, \\ Faculty of Medicine, University of Córdoba, Córdoba, Spain
}

\section{Key Words}

Natural killer cells $\cdot$ Melanoma $\cdot$ NKG2D $\cdot$ Natural

cytotoxicity receptors $\cdot$ DNAX accessory molecule-1

\begin{abstract}
The role of natural killer (NK) cells in tumor immunosurveillance has been recently underlined. A better understanding of the receptor-ligand interactions between NK cells and solid tumor cells is essential for introducing more effective NK cell-based immunotherapy protocols into clinical practice. We previously analyzed the surface expression of ligands for NK cell-activating receptors and costimulatory molecules in a large panel of melanoma cell lines. Although the expression of ligands for NK cell-activating receptors is variable, the majority of melanoma cell lines express ligands for NKG2D and for DNAX accessory molecule-1 (DNAM-1). While the NKG2D receptor has been described as the principal entity responsible for the lysis of several melanoma cell lines, the role of natural cytotoxicity receptors (NCRs) and DNAM-1 receptors in NK cell recognition and killing of melanoma cells has been recently emphasized. Antibody-mediated masking of NKG2D, NCRs, and DNAM-1 has proven that NKG2D, NCRs,
\end{abstract}

and DNAM-1 frequently cooperate in the lysis of melanoma cells. In this work, we provide an overview of recent advances in the study of melanoma cells' susceptibility to NK cellmediated lysis and how multiple receptor-ligand interactions participate in melanoma cell elimination.

Copyright $\odot 2011$ S. Karger AG, Basel

\section{Introduction}

Natural killer (NK) cells are potent effector cells that, unlike $\mathrm{T}$ lymphocytes, are able to eliminate target cells spontaneously without previous sensitization. NK cells are characterized by strong cytolytic activity against virus-infected cells and tumor cells $[1,2]$. After activation, NK cells can exert an immunomodulatory effect by the production of cytokines such as interferon (IFN)- $\gamma$ and tumor necrosis factor (TNF)- $\alpha$ or they can directly kill target cells by releasing perforins and granzymes [3]. In

Sara Morgado and Beatriz Sanchez-Correa contributed equally to this paper.

\section{KARGER \\ Fax +41613061234 \\ E-Mail karger@karger.ch}

www.karger.com (c) $2011 \mathrm{~S}$. Karger AG, Basel

$1662-811 X / 11 / 0034-0365 \$ 38.00 / 0$

Accessible online at:

www.karger.com/jin
Dr. Raquel Tarazona

Immunology Unit, Department of Physiology

University of Extremadura

ES-10003 Cáceres (Spain)

Tel. +34927 257 000, E-Mail rtarazon@unex.es 
addition, crosstalk between NK cells and dendritic cells (DCs) that results in NK cell activation and DC maturation has been described. NK cells promote the maturation of DCs and increase their capacity to produce proinflammatory cytokines and to stimulate $\mathrm{T}$ cell responses. In addition, activated NK cells kill DCs that have failed to undergo complete maturation [4].

Target susceptibility or resistance to NK-mediated lysis largely depends on the level of major histocompatibility complex (MHC) class I expression. Thus, NK cells selectively lyse cells that have low levels of MHC class I expression or have lost the expression of 1 or more MHC class I alleles. Viral infection or tumor transformation may induce the downregulation of MHC class I molecules and makes these cells potential targets for NK cells. The ability of NK cells to discriminate between self normal and transformed or infected target cells is due to the expression of MHC class I-specific inhibitory receptors $[5,6]$.

NK cells also express a large repertoire of activating receptors that sense cell stress, malignancy, and infection. The tune balance between signals transmitted through inhibitory and activating receptors upon interaction with their ligands on target cells regulates NK cells' effector function $[7,8]$.

There currently exists compelling in vitro and in vivo evidence of the contribution of NK cells in tumor immunosurveillance mediating the killing of different types of tumors. Clinical effects have been observed in hematopoietic stem cell transplantation and NK cell-based adoptive therapies $[9,10]$.

The possibility that the balance between inhibitory and activating NK receptor signals can be manipulated to increase the susceptibility of tumor cells to NK cellmediated lysis, together with recent advances in NK cell biology, have promoted translational research leading to clinical trials using different strategies to modulate NK cell function [11].

Here, we review recent advances in the study of the NK cell-mediated cytotoxicity of melanoma cells, with special focus on how multiple receptor-ligand interactions cooperate to achieve melanoma cell elimination.

\section{NK Cell-Mediated Recognition of Melanoma Cells}

Malignant melanoma is a very aggressive tumor originating from melanocytes. It is considered the most deadly form of skin cancer, frequently shows a tendency to spread, and rapidly progresses to a metastatic stage. Mel- anoma incidence has increased rapidly in the last decades [12]. The generally poor efficacy of chemotherapy and radiotherapy has redirected melanoma research to explore immune-based therapies [13, 14]. Melanoma cells can be recognized and eliminated by $\mathrm{CD} 8 \mathrm{~T}$ lymphocytes and NK cells as it has been shown that they can express not only tumor antigens but also ligands of NK-activating receptors. Whereas downregulation or complete loss of surface MHC class I molecules is a well-known mechanism used by melanoma cells to escape CD8 T cell surveillance $[15,16]$, it has been shown that the decreased expression of MHC class I molecules is associated with an increased susceptibility to NK cell-mediated lysis $[17,18]$. The capacity of NK cells to recognize and eliminate cells with a decreased expression of MHC class I molecules makes melanomas good targets for NK cell-based immunotherapy [19].

Freshly isolated resting NK cells display lower cytotoxic activity against melanoma cell lines than do interleukin (IL)-2-activated NK cells that efficiently kill both autologous $[18,20]$ and allogenic melanoma cell lines [20]. It has been suggested that resting NK cells require at least 2 receptor-ligand interactions to initiate the lysis of tumor cells; in contrast, IL-2-activated NK cells require only 1 trigger ligand to induce cytotoxicity [21,22]. In addition, IL-2-activated NK cells can express higher levels of activating receptors that contribute to the more efficient cytotoxicity observed [21].

Therefore, the susceptibility of melanoma cells to NK cell-mediated lysis depends on the expression of an adequate amount of ligands for NK cell-activating receptors and the level of expression of MHC class I molecules (fig. 1).

\section{Activating Receptors Involved in Melanoma-NK Cell Interactions}

Interactions between NK cells and melanoma cells have been extensively studied during the last decade. A better characterization of NK cell-activating receptors and the identification of some of their ligands has provided new insights in the analysis of NK cell recognition of melanoma. However, the wide range of NK cell-activating receptors makes it more difficult to discriminate the individual participation of the different NK cell receptors in the lysis of melanoma cells. Whereas an individual receptor may be involved in the lysis of some melanoma cell lines, simultaneous engagement of various receptors is required for the NK cell-mediated lysis of most 
Fig. 1. NK cell activation depends on the balance between inhibitory and activating signals transmitted through receptors on the surface of NK cells. a MHC class I molecules expressed by tumor cells interact with MHC class I-specific inhibitory receptors on the surface on NK cells and transmit inhibitory signals to avoid tumor cell elimination. $\mathbf{b}$ In the absence of inhibitory signals, the expression of ligands for activating receptors makes tumor cells susceptible to NK cell cytotoxicity. c The lysis of tumor cells expressing both MHC class I molecules and ligands for activating receptors depends on the tune balance of signals transmitted through inhibitory and activating NK cell receptors.

Fig. 2. The interaction between the activating receptors NKG2D, NCRs, and DNAM-1 on the surface of NK cells with their ligands on melanoma cells results in the activation of NK cells and the lysis of melanoma cells. The expression of high levels of ligands of NK major receptors, such as NKG2D (a), or NCRs (b) triggers NK cell cytotoxicity. Simultaneous engagement of several activating receptors (c) may be required for the lysis of melanoma cells expressing low levels of their ligands.
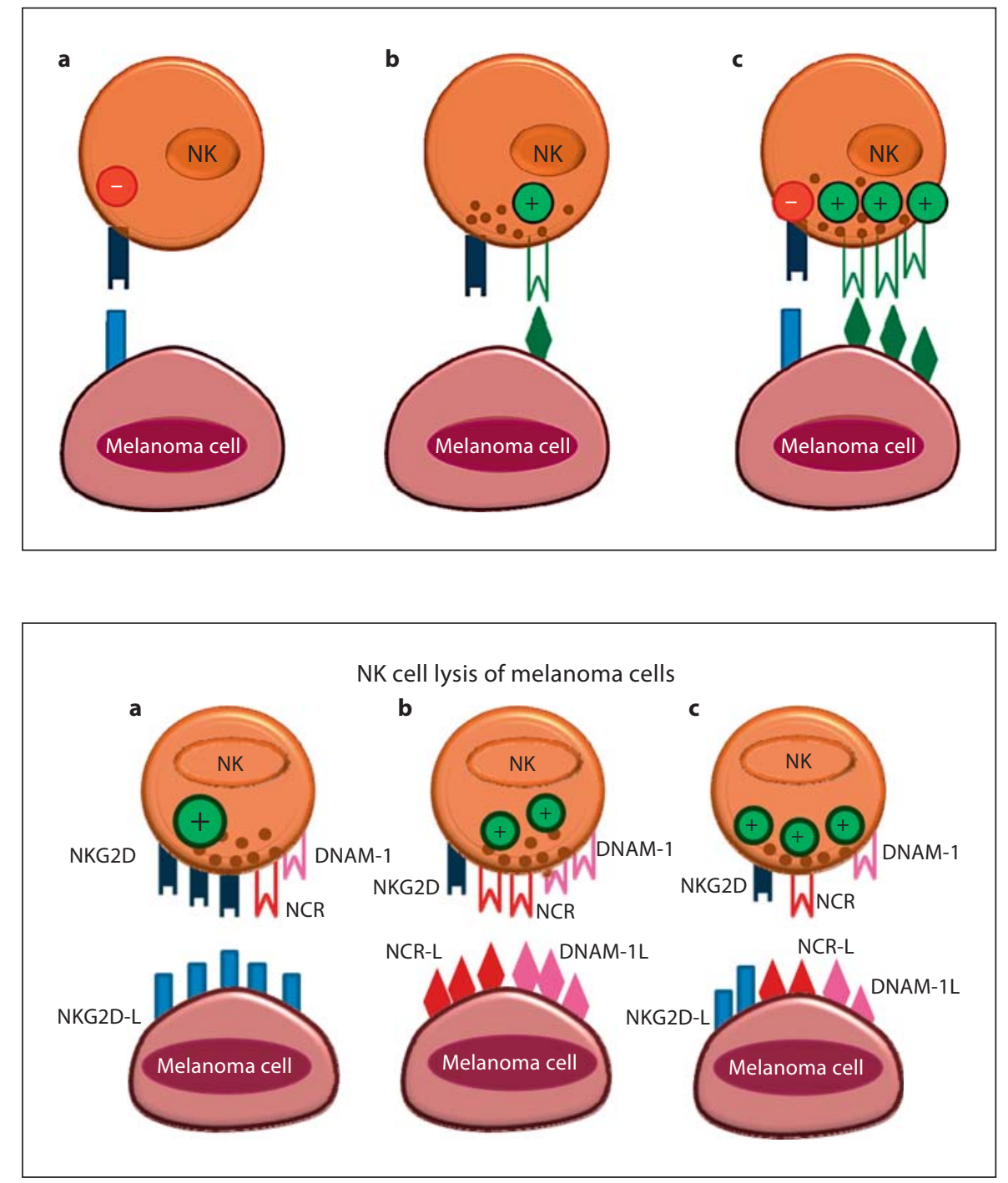

melanoma cell lines. Thus, collaboration between different receptors may help to explain the different findings regarding NK cell activation against melanoma cells (fig. 2).

\section{NKG2D and Its Ligands in Melanoma}

Human NKG2D is a C-type lectin-like receptor constitutively expressed on NK cells, CD $8 \alpha \beta$ T cells, $\gamma \delta \mathrm{T}$ cells, and most invariant NKT cells as well as in subsets of CD4 T cells in certain clinical conditions such as cancer or autoimmune diseases [23-25]. It is an activating receptor for NK cells, promoting both the lysis of target cells and cytokine release, and it acts as a costimulatory receptor for CD8 T cells. Human NKG2D forms homodimers, and signaling through this receptor requires its as- sociation with the adaptor molecule DAP10, which also stabilizes its surface expression $[25,26]$.

Ligands for NKG2D comprise the MHC class I chainrelated gen A (MICA) and gen B (MICB) proteins and UL-16 binding proteins 1-5 (ULBP1-5). NKG2D ligands can be upregulated under stressful conditions such as tumor transformation or infections $[25,26]$.

The implication of the activating receptor NKG2D and its ligands MICA/B and ULBPs in NK cell recognition and killing of melanoma cells has been extensively studied, albeit with some controversial results. Analysis of the expression of NKG2D ligands on melanoma cell lines showed that the majority expresses ligands for NKG2D, supporting its role as a major triggering receptor inducing the NK cell-mediated cytotoxicity of melanoma 
Fig. 3. Melanoma cells can escape NK immunosurveillance by downregulation (a) or shedding of ligands for NK cell-activating receptors (b) or by downregulation of the activating receptors (c). The 3 mechanisms can simultaneously be involved in melanoma evasion of NK cell-mediated killing.

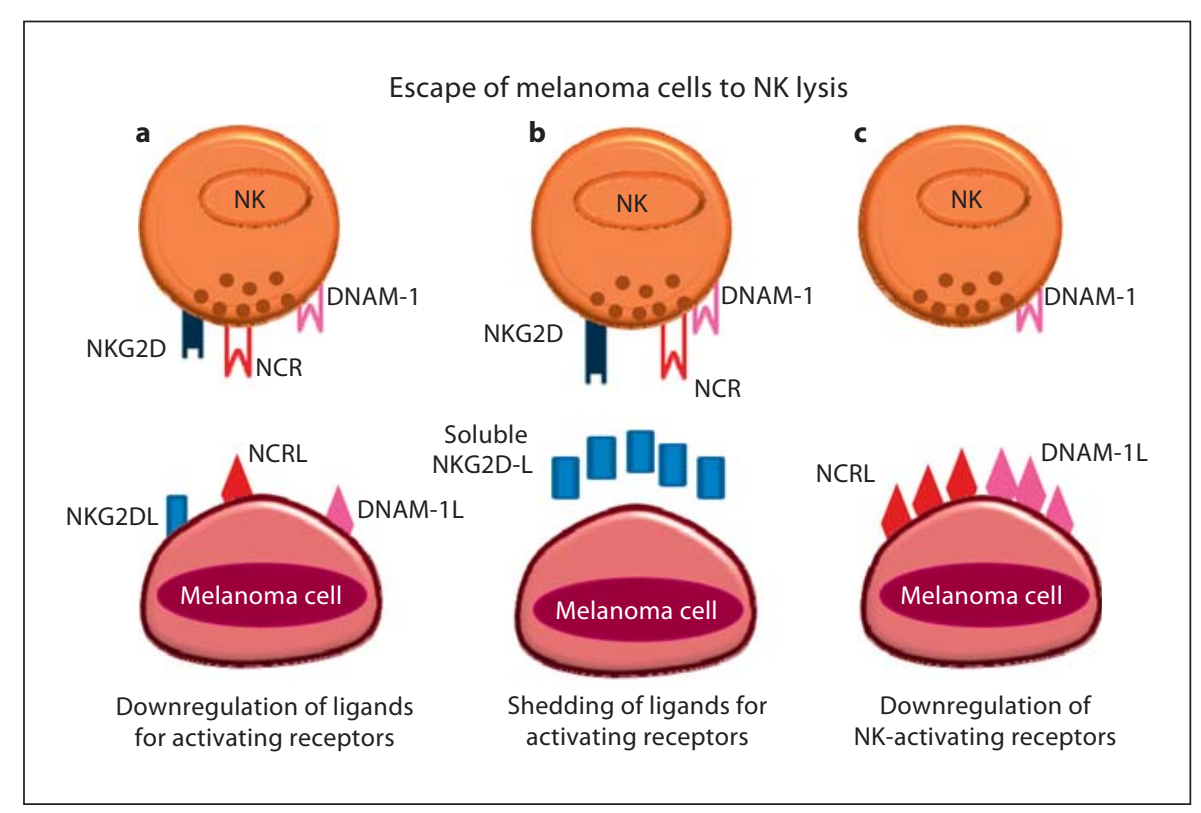

(fig. 2a). Pende et al. [27] analyzed melanoma cell lines by flow cytometry $(n=18)$ and showed that MICA was expressed in the majority of cell lines while MICB was not present. ULBPs were expressed in different patterns, and they may or may not be coexpressed with MICA. We recently analyzed the expression of ligands for the activating receptor NKG2D in a large panel of melanoma cell lines $(\mathrm{n}=124)$. Whereas MICA/B was expressed in $80 \%$ of melanoma cell lines, ULBP expression was less frequent (ULBP1, 15\%; ULBP2, 25\%, and ULBP3, 20\%) [28]. Using immunohistochemistry, Vetter et al. [29] found that $77.5 \%$ of primary tumors $(n=40)$ and $65 \%$ of metastatic melanomas $(n=20)$ expressed MICA/B. The staining pattern showed considerable variations regarding intensity and frequency. MICA/B was not expressed on benign melanocytic lesions, suggesting that its expression is associated with tumor transformation [29]. However, in another study, Maccalli et al. [30] analyzed the expression of ULBPs and MICA in primary melanomas and metastatic lesions and showed that only primary tumors expressed a significant level of NKG2D ligands. It is also interesting to note that in uveal melanoma $50 \%$ of primary tumors expressed MICA/B but none of the metastatic lesions did [31]. Altogether, these results suggest that, along with melanoma progression, decreasing expression of MICA/B and ULBPs by tumor cells may occur as a consequence of immune selection pressure against MICA/B-positive melanoma cells (fig. 3a).
A correlation between the expression of NKG2D ligands on target cells and the NK cell-mediated lysis of melanoma cells has been shown, suggesting that NKG2Dmediated NK cell triggering is likely involved in the lysis of melanoma cells. In melanoma cell lines that coexpress MICA and ULBPs, both molecules participate in NK cell activation via NKG2D [27]. Blocking experiments of the NKG2D receptor also highlight the role of NKG2D as a major receptor against melanoma cells when they express NKG2D ligands [28]. In addition, analysis of the individual participation of NKG2D was performed using the C1R cell line that is resistant to NK cell-mediated lysis. Transfection of C1R cells with ULBP-1 makes them susceptible to NK cell cytotoxicity, and a direct correlation between the expression level of NKG2D on NK cells and the NKG2D-mediated killing of C1R cells transfected with ULBP-1 was observed [32].

Melanoma cells can evade NKG2D-mediated recognition by innate and adaptive immunity using different strategies [20]. Thus, shedding of NKG2D ligands MICA and ULBP-2 has been detected in serum from melanoma patients and interestingly high levels of these molecules are associated with a reduced overall survival [33] (fig. 3b).

NKG2D ligand expression can be modulated by several cytokines. IFN- $\gamma$ induces a decrease in the expression of MICA and, in some cases, ULBP-2 that is dependent on the cytokine dose and the duration of treatment, making IFN- $\gamma$ treated melanoma cells less susceptible to NKG2D-mediated NK cell cytotoxicity [34]. IL-10 also
Morgado/Sanchez-Correa/Casado/ Duran/Gayoso/Labella/Solana/Tarazona 
induces the downregulation of MICA and ULBP-2 expression on several melanoma cell lines, whereas MHC class I expression does not change. This reduction in the expression of NKG2D ligands results in a diminished susceptibility to NK-mediated lysis [35]. In line with these results, it has been described in melanoma patients that higher serum levels of IL-10 are associated with a worse prognosis [36] that could be related to the downregulation of NKG2D ligands in melanoma cells.

Finally, it is of interest to note that the decreased expression of NKG2D found on NK cells from metastatic melanoma patients correlates with a lower NK cell cytotoxicity [37], supporting the relevant role of NKG2D in NK killing of melanoma (fig. 3c).

\section{Natural Cytotoxicity Receptors and Their Ligands in}

\section{Melanoma}

Natural cytotoxicity receptors (NCRs) represent key triggering receptors involved in the lysis of tumor and virus-infected cells. They belong to the immunoglobulin superfamily of receptors and 3 members have been identified: NKp30 and NKp46 that are constitutively expressed on NK cells and NKp44 that is induced after activation [38]. NCRs associate with CD3 $\zeta$ or FceRI- $\gamma$ molecules containing immunoreceptor tyrosine-based activation motifs (ITAMs) that mediate the transduction of the triggering signal [7].

Whereas several viral NCR ligands such as viral hemagglutinins or the main tegument protein (pp65) of human cytomegalovirus have been identified, their cellular ligands are still poorly characterized [2]. Two cellular ligands have so far been identified on tumor cells: heparan sulfate, recognized by all NCRs [39], and B7-H6, a member of the B7 family and a ligand for NKp30 [40].

The contribution of NCRs to melanoma cell recognition and killing by NK cells shows that NKp46 has a central role in mediating the lysis of several target cells, with a correlation between the surface density of NKp46 on NK cells and target cell lysis [41, 42]. Antibody-mediated blocking experiments showed that NKp30 is a major receptor involved in the lysis of some melanoma cell lines and may cooperate with NKp46 and/or NKp44, depending on the expression of the appropriate ligands by the target cell [43], supporting the involvement of several NCRs in melanoma recognition and killing.

Due to limited knowledge regarding cell ligands for NCRs, their expression in melanoma has been analyzed using either fusion proteins containing the extracellular domain of NKp46, NKp30, or NKp44 fused to an aminoterminal isoleucine zipper (NCR-ILZ) or fused to the Fc domain of IgG (NCR-Fc). Melanoma cell lines can express ligands for NKp30 and NKp44 [44] and NKp46 [45]. The analysis of melanoma cells from patients with metastatic melanomas showed that NKp44 ligands were present in all melanoma samples analyzed, NKp30 ligands appeared in the majority of samples, and none expressed ligands for NKp46 [32]. In another study [46], the analysis of a limited number of paired samples showed staining with NKp44-Fc and NKp46-Fc on melanoma cells from lymph nodes, whereas negative or very low staining was found on melanoma cells obtained from skin metastases. Since lymph node metastases were reported to precede the appearance of skin metastases, the different pattern of expression of NCR-Fc chimeras in melanoma cell lines may reflect diverse stages in disease progression. The staining pattern correlated with the higher susceptibility of lymph node-derived melanoma cell lines to NK cell recognition and killing [46]. It has been suggested that NCR ligands are overexpressed in early stages of the disease and that tumor variants with lower levels of NCR ligands may occur during melanoma progression $[45,46]$ (fig. 3a).

Interestingly, the expression of NKp30 was significantly decreased on NK cells from metastatic melanoma patients and the decrease was associated with a reduced ability to kill melanoma cells [32] (fig. 3c).

\section{DNAX Accessory Molecule-1 and Its Ligands in Melanoma}

Another NK cell-triggering receptor of the immunoglobulin superfamily is DNAX accessory molecule-1 (DNAM-1); in humans it is expressed on NK cells, T cells, monocytes, a subset of B cells, and platelets. DNAM-1 ligands are CD155 (poliovirus receptor) and CD112 (nectin-2) [47-49]. CD155 and CD112 can be expressed on different types of tumors including melanoma, leukemia, and ovarian carcinoma [28, 48, 50-52]. Stimulation of DNAM-1 by its interaction with its ligands leads to NK cell activation and target cell lysis [47-49].

The role of DNAM-1 in the recognition of melanoma cells is highlighted by the frequent expression of its ligands CD155 and CD112 [28, 46, 48]. Our results showed that DNAM-1 ligands were frequently expressed by melanoma cell lines, in particular CD155 which was present in the majority of the cell lines, in contrast with CD112 which was found only on $26 \%$ of the lines [28]. These results differ from those obtained in other tumor cells such as leukemic blasts where the expression of CD112 was more frequent than that of CD155 [52]. DNAM-1 participates in the NK cell-mediated lysis of some melanoma 
cells, but collaboration with NCRs is frequently required $[47,48]$.

The role of DNAM-1 in the control of tumor metastases has been recently explored using cell lines from different lineages. It has been shown that DNAM-1 is required for the lysis of poorly immunogenic melanoma metastases lacking ligands for NKG2D [53]. DNAM-1 is a costimulatory molecule for CD8 T cells when nonprofessional antigen-presenting cells are used for stimulation. Similarly, NK cells require DNAM-1 for the elimination of tumor cells that are less susceptible to NK cellmediated cytotoxicity due to the low expression of ligands for other NK cell-activating receptors [54]. DNAM-1 interaction with its ligands has also been shown to be critical in NK cell recognition of ovarian carcinoma cells and blasts in myelodysplastic syndrome [50, 51]. Its role in tumor immunosurveillance has been recently demonstrated in vivo using a DNAM-1 knockout mice model [54, 55].

\section{Cooperation between Different Activating Receptors in} Melanoma

The model of NK cell activation against tumors proposed by Bottino et al. [56] suggests that the ligands recognized by major receptors, such as NKG2D and NCR, are expressed mainly by tissues undergoing different kinds of cellular stress such as tumor transformation or viral infection. Engagement of these receptors by their ligands results in potent NK cell activation that in some instances might even override signaling mediated by inhibitory receptors. In order to achieve full activation of NK cells, other activating or costimulatory receptors may require overexpression of their ligands or simultaneous signaling by several activating receptors in combination with the absence of inhibitory signals mediated by MHC class I-specific receptors [56].

The results reviewed here support the notion that this model of NK cell activation is applicable to NK-melanoma cell interaction (fig. 2c). Thus, Markel et al. [32] showed a predominant role of NKG2D, NKp30, and NKp44 in the recognition and lysis of melanoma cells. The specific involvement and additive effect of NKG2D and NKp30 was evident by the partial abolishment of cytotoxic activity in the presence of blocking monoclonal antibodies, but they also have residual killing activity probably enabled via NKp44 [32].

Similar to the downregulation of activating NK cell receptors observed in leukemia patients [52, 57, 58], a decreased expression of NCRs was observed on NK cells from metastatic melanoma patients as compared to healthy donors. This observation is probably a melanoma-driven effect, although it cannot be excluded that individuals with a natural lower expression of NKp30 are more susceptible to the development of melanoma [32].

In another study, analysis of melanoma cells that expressed ligands for NKp46, NKp44, and DNAM-1 but very low levels of NKG2D ligands showed that these melanoma cells were recognized and eliminated in an NCRand DNAM-1-dependent fashion without the implication of NKG2D [46].

Altogether, the results obtained by different groups indicate that in most instances NK cell-mediated recognition and lysis of melanoma cell lines requires cooperation between different activating receptors (fig. 2c). According to evidence from in vitro studies, melanoma cells obtained ex vivo from melanoma patients show high variability in the expression of ligands for NKG2D and NCR receptors that may be the consequence of differences in tumor progression stage, tumor localization, or immune selection pressure. This heterogeneity in the expression of ligands may explain the discrepancies observed by different groups regarding the role of different activating receptors in the rejection of melanoma. In addition, studies on the expression of NKG2D or NCRs on NK cells from melanoma patients have shown that they can be decreased, likely as a consequence of their interaction with their ligands either on the tumor cell surface, or shed by the melanoma cells.

\section{Future Perspectives and Concluding Remarks}

Melanoma cells can be recognized by NK cells through different activating receptors, but their lysis may require different thresholds of NK cell activation, depending on the expression level and the presence of ligands for different activating receptors. Thus, the participation of different activating receptors may act in a synergistic manner favoring the recognition and elimination of melanoma cells.

The enhancement of activating receptors on NK cells from tumor patients may give rise to new therapeutic approaches. Modulation of the expression of NK cell-activating receptors by IL-15 has been recently shown. NK cells obtained from acute myeloid patients showed reduced expression of activating NK cell receptors. When these NK cells were cultured with IL-15, the expression of the activating receptors NKp30, NKp46, and NKG2D was significantly increased and resulted in a higher cytotoxicity against autologous leukemic blasts [59]. These re- 
sults open new possibilities for the treatment of tumor patients by enhancing the capability of NK cells to kill tumor cells.

In conclusion, $\mathrm{NK}$ cell-activating receptor expression directly correlates with NK-mediated killing of tumor cells. Melanoma cells are mainly recognized by NKG2D, NCRs, and DNAM-1, and several alterations in the expression of these receptors can be found in cancer patients. The expression of ligands for different NK cellactivating receptors is variable on different melanoma cells and can be used as an indicator of NK cell susceptibility. Simultaneous engagement of several activating receptors contributes to efficient NK cell-mediated lysis of melanoma cells. Thus, for the use of NK cells in immunotherapy against melanoma, phenotypic characterization of melanoma cells will be useful for predicting the therapeutic efficacy of NK cells. New therapeutic strategies aimed at increasing the expression of ligands for NKactivating receptors and at modulating the expression of NK cell-activating receptors may contribute to the development of more effective therapy for individual patients.

\section{Acknowledgements}

We apologize to our colleagues whose work was not cited due to space limitations. This work was supported by grants to R.T. (SAF2006-03687 and SAF2009-09711 from the Ministry of Science and Innovation of Spain), by PRI09A029 and grants to the INPATT research group from the Junta de Extremadura (GRU09156 and GRU10104) and from the University of Extremadura, cofinanced by European Regional Development Funds (FEDER), and by grants to R.S. (PS09/00723 from the Spanish Ministry of Health and JA0292/07 from the Junta de Andalucía). S.M. is a predoctoral fellow supported by grant PRE07047 from the Junta de Extremadura. B.S.C. is a postdoctoral fellow supported by grant ACCIII/22 from the University of Extremadura. I.G. is a postdoctoral fellow supported by the Spanish Network for Research in Infectious Diseases (REIPI RD06/0008), cofinanced by FEDER. We thank J.J. Gordillo and M.R. Gonzalez for their technical assistance.

\section{References}

1 Brandstadter JD, Yang Y: Natural killer cell responses to viral infection. J Innate Immun 2011;3:274-279.

-2 Arnon TI, Markel G, Mandelboim O: Tumor and viral recognition by natural killer cells receptors. Semin Cancer Biol 2006;16:348358.

- 3 Farag SS, Caligiuri MA: Human natural killer cell development and biology. Blood Rev 2006;20:123-137.

4 Wehner R, Dietze K, Bachmann M, Schmitz $\mathrm{M}$ : The bidirectional crosstalk between human dendritic cells and natural killer cells. J Innate Immun 2011;3:258-263.

5 Ljunggren HG, Karre K: In search of the 'missing self': MHC molecules and NK cell recognition. Immunol Today 1990;11:237244.

-6 Long EO: Negative signaling by inhibitory receptors: the NK cell paradigm. Immunol Rev 2008;224:70-84.

7 Bryceson YT, Long EO: Line of attack: NK cell specificity and integration of signals. Curr Opin Immunol 2008;20:344-352.

-8 Yokoyama WM, Altfeld M, Hsu KC: Natural killer cells: tolerance to self and innate immunity to viral infection and malignancy. Biol Blood Marrow Transplant 2010;16:S97S105.

NK Cell-Mediated Lysis of Melanoma
-9 Malmberg KJ, Bryceson YT, Carlsten M, Andersson S, Bjorklund A, Bjorkstrom NK, Baumann BC, Fauriat C, Alici E, Dilber MS, Ljunggren HG: NK cell-mediated targeting of human cancer and possibilities for new means of immunotherapy. Cancer Immunol Immunother 2008;57:1541-1552.

10 Nguyen S, Beziat V, Roos-Weil D, Vieillard $\mathrm{V}$ : Role of natural killer cells in hematopoietic stem cell transplantation: myth or reality? J Innate Immun 2011, E-pub ahead of print.

11 Terme M, Ullrich E, Delahaye NF, Chaput N, Zitvogel L: Natural killer cell-directed therapies: moving from unexpected results to successful strategies. Nat Immunol 2008;9:486494.

12 Lee JA: The determination of melanoma stage at diagnosis. Dermatol Res Pract 2010; 839829:1-3.

13 Erdmann MK: Immunity unleashed in melanoma. Lancet Oncol 2010;11:108-109.

14 Berry LJ, Moeller M, Darcy PK: Adoptive immunotherapy for cancer: the next generation of gene-engineered immune cells. Tissue Antigens 2009;74:277-289.

15 Garrido F, Algarra I, Garcia-Lora AM: The escape of cancer from $\mathrm{T}$ lymphocytes: immunoselection of MHC class I loss variants harboring structural-irreversible 'hard' lesions. Cancer Immunol Immunother 2010; 59:1601-1606.
16 Mendez R, Aptsiauri N, Del CA, Maleno I, Cabrera T, Ruiz-Cabello F, Garrido F, Garcia-Lora A: HLA and melanoma: multiple alterations in HLA class I and II expression in human melanoma cell lines from ESTDAB cell bank. Cancer Immunol Immunother 2009;58:1507-1515.

17 Pende D, Accame L, Pareti L, Mazzocchi A, Moretta A, Parmiani G, Moretta L: The susceptibility to natural killer cell-mediated lysis of HLA class I-positive melanomas reflects the expression of insufficient amounts of different HLA class I alleles. Eur J Immunol 1998;28:2384-2394.

18 Carrega P, Pezzino G, Queirolo P, Bonaccorsi I, Falco M, Vita G, Pende D, Misefari A, Moretta A, Mingari MC, Moretta L, Ferlazzo G: Susceptibility of human melanoma cells to autologous natural killer (NK) cell killing: HLA-related effector mechanisms and role of unlicensed NK cells. PLoS One 2009; 4:e8132.

19 Burke S, Lakshmikanth T, Colucci F, Carbone E: New views on natural killer cellbased immunotherapy for melanoma treatment. Trends Immunol 2010;31:339-345.

20 Solana R, Casado JG, Delgado E, DelaRosa O, Marin J, Duran E, Pawelec G, Tarazona R: Lymphocyte activation in response to melanoma: interaction of NK-associated receptors and their ligands. Cancer Immunol Immunother 2007;56:101-109. 
-21 Bryceson YT, March ME, Ljunggren HG, Long EO: Activation, coactivation, and costimulation of resting human natural killer cells. Immunol Rev 2006;214:73-91.

$\checkmark 22$ North J, Bakhsh I, Marden C, Pittman H, Addison E, Navarrete C, Anderson R, Lowdell MW: Tumor-primed human natural killer cells lyse NK-resistant tumor targets: evidence of a two-stage process in resting NK cell activation. J Immunol 2007;178:8594.

-23 Peralbo E, DelaRosa O, Gayoso I, Pita ML, Tarazona R, Solana R: Decreased frequency and proliferative response of invariant Valpha24Vbeta11 natural killer T (iNKT) cells in healthy elderly. Biogerontology 2006; 7: 483-492.

24 Champsaur M, Lanier LL: Effect of NKG2D ligand expression on host immune responses. Immunol Rev 2010;235:267-285.

-25 Burgess SJ, Maasho K, Masilamani M, Narayanan S, Borrego F, Coligan JE: The NKG2D receptor: immunobiology and clinical implications. Immunol Res 2008;40:1834.

26 Waldhauer I, Steinle A: NK cells and cancer immunosurveillance. Oncogene 2008;27: 5932-5943.

27 Pende D, Rivera P, Marcenaro S, Chang CC, Biassoni R, Conte R, Kubin M, Cosman D, Ferrone S, Moretta L, Moretta A: Major histocompatibility complex class I-related chain A and UL16-binding protein expression on tumor cell lines of different histotypes: analysis of tumor susceptibility to NKG2D-dependent natural killer cell cytotoxicity. Cancer Res 2002;62:6178-6186.

-28 Casado JG, Pawelec G, Morgado S, SanchezCorrea B, Delgado E, Gayoso I, Duran E, Solana R, Tarazona R: Expression of adhesion molecules and ligands for activating and costimulatory receptors involved in cell-mediated cytotoxicity in a large panel of human melanoma cell lines. Cancer Immunol Immunother 2009;58:1517-1526.

-29 Vetter CS, Groh V, Thor SP, Spies T, Brocker EB, Becker JC: Expression of stress-induced MHC class I related chain molecules on human melanoma. J Invest Dermatol 2002;118: 600-605.

-30 Maccalli C, Nonaka D, Piris A, Pende D, Rivoltini L, Castelli C, Parmiani G: NKG2Dmediated antitumor activity by tumor-infiltrating lymphocytes and antigen-specific Tcell clones isolated from melanoma patients. Clin Cancer Res 2007;13:7459-7468.

- 31 Vetter CS, Lieb W, Brocker EB, Becker JC: Loss of nonclassical MHC molecules MIC$\mathrm{A} / \mathrm{B}$ expression during progression of uveal melanoma. Br J Cancer 2004;91:1495-1499.

- 32 Markel G, Seidman R, Besser MJ, Zabari N, Ortenberg R, Shapira R, Treves AJ, Loewenthal R, Orenstein A, Nagler A, Schachter J: Natural killer lysis receptor (NKLR)/NKLRligand matching as a novel approach for enhancing anti-tumor activity of allogeneic NK cells. PLoS One 2009;4:e5597.
33 Paschen A, Sucker A, Hill B, Moll I, Zapatka M, Nguyen XD, Sim GC, Gutmann I, Hassel J, Becker JC, Steinle A, Schadendorf D, Ugurel S: Differential clinical significance of individual NKG2D ligands in melanoma: soluble ULBP2 as an indicator of poor prognosis superior to S100B. Clin Cancer Res 2009; 15:5208-5215.

34 Schwinn N, Vokhminova D, Sucker A, Textor S, Striegel S, Moll I, Nausch N, Tuettenberg J, Steinle A, Cerwenka A, Schadendorf $D$, Paschen A: Interferon-gamma down-regulates NKG2D ligand expression and impairs the NKG2D-mediated cytolysis of MHC class I-deficient melanoma by natural killer cells. Int J Cancer 2009;124:1594-1604.

35 Serrano AE, Menares-Castillo E, GarridoTapia M, Ribeiro CH, Hernandez CJ, Mendoza-Naranjo A, Gatica-Andrades M, Valenzuela-Diaz R, Zuniga R, Lopez MN, Salazar-Onfray F, Aguillon JC, Molina MC: Interleukin 10 decreases MICA expression on melanoma cell surface. Immunol Cell Biol 2011;89:447-457.

-36 Torisu-Itakura H, Lee JH, Huynh Y, Ye X, Essner R, Morton DL: Monocyte-derived IL10 expression predicts prognosis of stage IV melanoma patients. J Immunother 2007;30: 831-838.

37 Konjevic G, Mirjacic MK, Jurisic V, Babovic $\mathrm{N}$, Spuzic I: Biomarkers of suppressed natural killer (NK) cell function in metastatic melanoma: decreased NKG2D and increased CD158a receptors on CD3-CD16+ NK cells. Biomarkers 2009;14:258-270.

- 38 Moretta L, Biassoni R, Bottino C, Mingari MC, Moretta A: Human NK-cell receptors. Immunol Today 2000;21:420-422.

39 Hecht ML, Rosental B, Horlacher T, Hershkovitz O, De Paz JL, Noti C, Schauer S, Porgador A, Seeberger PH: Natural cytotoxicity receptors NKp30, NKp44 and NKp46 bind to different heparan sulfate/heparin sequences. J Proteome Res 2009;8:712-720.

40 Brandt CS, Baratin M, Yi EC, Kennedy J, Gao Z, Fox B, Haldeman B, Ostrander CD, Kaifu T, Chabannon C, Moretta A, West R, Xu W, Vivier E, Levin SD: The B7 family member B7-H6 is a tumor cell ligand for the activating natural killer cell receptor $\mathrm{NKp} 30 \mathrm{in} \mathrm{hu-}$ mans. J Exp Med 2009;206:1495-1503.

41 Sivori S, Pende D, Bottino C, Marcenaro E, Pessino A, Biassoni R, Moretta L, Moretta A: $\mathrm{NKp} 46$ is the major triggering receptor involved in the natural cytotoxicity of fresh or cultured human NK cells: correlation between surface density of NKp46 and natural cytotoxicity against autologous, allogeneic or xenogeneic target cells. Eur J Immunol 1999;29:1656-1666.

42 Pessino A, Sivori S, Bottino C, Malaspina A, Morelli L, Moretta L, Biassoni R, Moretta A: Molecular cloning of NKp46: a novel member of the immunoglobulin superfamily involved in triggering of natural cytotoxicity. J Exp Med 1998;188:953-960.
43 Pende D, Parolini S, Pessino A, Sivori S, Augugliaro R, Morelli L, Marcenaro E, Accame L, Malaspina A, Biassoni R, Bottino C, Moretta L, Moretta A: Identification and molecular characterization of NKp30, a novel triggering receptor involved in natural cytotoxicity mediated by human natural killer cells. J Exp Med 1999;190:1505-1516.

44 Byrd A, Hoffmann SC, Jarahian M, Momburg F, Watzl C: Expression analysis of the ligands for the natural killer cell receptors NKp30 and NKp44. PLoS One 2007;2:e1339.

45 Cagnano E, Hershkovitz O, Zilka A, Bar-Ilan A, Golder A, Sion-Vardy N, Bogdanov-Berezovsky A, Mandelboim O, Benharroch D, Porgador A: Expression of ligands to NKp46 in benign and malignant melanocytes. J Invest Dermatol 2008;128:972-979.

46 Lakshmikanth T, Burke S, Ali TH, Kimpfler S, Ursini F, Ruggeri L, Capanni M, Umansky V, Paschen A, Sucker A, Pende D, Groh V, Biassoni R, Hoglund P, Kato M, Shibuya K, Schadendorf D, Anichini A, Ferrone S, Velardi A, Karre K, Shibuya A, Carbone E, Colucci F: NCRs and DNAM-1 mediate NK cell recognition and lysis of human and mouse melanoma cell lines in vitro and in vivo. J Clin Invest 2009;119:1251-1263.

47 Bottino C, Castriconi R, Pende D, Rivera P, Nanni M, Carnemolla B, Cantoni C, Grassi J, Marcenaro S, Reymond N, Vitale M, Moretta L, Lopez M, Moretta A: Identification of PVR (CD155) and Nectin-2 (CD112) as cell surface ligands for the human DNAM1 (CD226) activating molecule. J Exp Med 2003;198:557-567.

- 48 Pende D, Bottino C, Castriconi R, Cantoni C, Marcenaro S, Rivera P, Spaggiari GM, Dondero A, Carnemolla B, Reymond N, Mingari MC, Lopez M, Moretta L, Moretta A: PVR (CD155) and Nectin-2 (CD112) as ligands of the human DNAM-1 (CD226) activating receptor: involvement in tumor cell lysis. Mol Immunol 2005;42:463-469.

49 Tahara-Hanaoka S, Shibuya K, Onoda Y, Zhang H, Yamazaki S, Miyamoto A, Honda S, Lanier LL, Shibuya A: Functional characterization of DNAM-1 (CD226) interaction with its ligands PVR (CD155) and nectin-2 (PRR-2/CD112). Int Immunol 2004; 16:533538.

50 Carlsten M, Baumann BC, Simonsson M, Jadersten M, Forsblom AM, Hammarstedt C, Bryceson YT, Ljunggren HG, HellstromLindberg E, Malmberg KJ: Reduced DNAM1 expression on bone marrow NK cells associated with impaired killing of CD34+ blasts in myelodysplastic syndrome. Leukemia 2010;24:1607-1616.

51 Carlsten M, Norell H, Bryceson YT, Poschke I, Schedvins K, Ljunggren HG, Kiessling R, Malmberg KJ: Primary human tumor cells expressing CD155 impair tumor targeting by down-regulating DNAM-1 on NK cells. J Immunol 2009;183:4921-4930. 
52 Sanchez-Correa B, Gayoso I, Bergua JM, Casado JG, Morgado S, Solana R, Tarazona R: Decreased expression of DNAM-1 on NK cells from acute myeloid leukemia patients. Immunol Cell Biol 2011, E-pub ahead of print.

53 Chan CJ, Andrews DM, McLaughlin NM, Yagita H, Gilfillan S, Colonna M, Smyth MJ: DNAM-1/CD155 interactions promote cytokine and NK cell-mediated suppression of poorly immunogenic melanoma metastases. J Immunol 2010;184:902-911.
4 Gilfillan S, Chan CJ, Cella M, Haynes NM, Rapaport AS, Boles KS, Andrews DM, Smyth MJ, Colonna M: DNAM-1 promotes activation of cytotoxic lymphocytes by nonprofessional antigen-presenting cells and tumors. J Exp Med 2008;205:2965-2973.

55 Iguchi-Manaka A, Kai H, Yamashita Y, Shibata K, Tahara-Hanaoka S, Honda S, Yasui T, Kikutani H, Shibuya K, Shibuya A: Accelerated tumor growth in mice deficient in DNAM-1 receptor. J Exp Med 2008;205: 2959-2964.

56 Bottino C, Moretta L, Moretta A: NK cell activating receptors and tumor recognition in humans. Curr Top Microbiol Immunol 2006;298:175-182.

57 Costello RT, Sivori S, Marcenaro E, LafagePochitaloff M, Mozziconacci MJ, Reviron D, Gastaut JA, Pende D, Olive D, Moretta A: Defective expression and function of natural killer cell-triggering receptors in patients with acute myeloid leukemia. Blood 2002;99: 3661-3667.
8 Fauriat C, Just-Landi S, Mallet F, Arnoulet C, Sainty D, Olive D, Costello RT: Deficient expression of NCR in NK cells from acute myeloid leukemia: evolution during leukemia treatment and impact of leukemia cells in NCRdull phenotype induction. Blood 2007; 109:323-330.

59 Szczepanski MJ, Szajnik M, Welsh A, Foon KA, Whiteside TL, Boyiadzis M: Interleukin-15 enhances natural killer cell cytotoxicity in patients with acute myeloid leukemia by upregulating the activating NK cell receptors. Cancer Immunol Immunother 2010;59: 73-79. 